\title{
Metformin alleviates muscle wasting post- thermal injury by increasing Pax7-positive muscle progenitor cells
}

\author{
Yusef Yousuf ${ }^{1}$, Andrea Datu', Ben Barnes ${ }^{1}$, Saeid Amini-Nik ${ }^{1,2,3^{*}}$ and Marc G. Jeschke $e^{1,3,4,5^{*}}$ (D)
}

\begin{abstract}
Background: Profound skeletal muscle wasting and weakness is common after severe burn and persists for years after injury contributing to morbidity and mortality of burn patients. Currently, no ideal treatment exists to inhibit muscle catabolism. Metformin is an anti-diabetic agent that manages hyperglycemia but has also been shown to have a beneficial effect on stem cells after injury. We hypothesize that metformin administration will increase protein synthesis in the skeletal muscle by increasing the proliferation of muscle progenitor cells, thus mitigating muscle atrophy post-burn injury.

Methods: To determine whether metformin can attenuate muscle catabolism following burn injury, we utilized a $30 \%$ total burn surface area (TBSA) full-thickness scald burn in mice and compared burn injuries with and without metformin treatment. We examined the gastrocnemius muscle at 7 and 14 days post-burn injury.
\end{abstract}

Results: At 7 days, burn injury significantly reduced myofiber cross-sectional area (CSA) compared to sham, $p<0.05$. Metformin treatment significantly attenuated muscle catabolism and preserved muscle CSA at the sham size. To investigate metformin's effect on satellite cells (muscle progenitors), we examined changes in Pax7, a transcription factor regulating the proliferation of muscle progenitors. Burned animals treated with metformin had a significant increase in Pax7 protein level and the number of Pax7-positive cells at 7 days post-burn, $p<0.05$. Moreover, through BrdU proliferation assay, we show that metformin treatment increased the proliferation of satellite cells at 7 days post-burn injury, $p<0.05$.

Conclusion: In summary, metformin's various metabolic effects and its modulation of stem cells make it an attractive alternative to mitigate burn-induced muscle wasting while also managing hyperglycemia.

Keywords: Metformin, Skeletal muscle, Muscle wasting, Thermal injury, Burn, Satellite cells, Pax7, Fat infiltration

\section{Introduction}

Burn injury results in a debilitating stress response termed the hypermetabolic response resulting in profound changes to several organ systems. Despite recent advances in therapeutic strategies such as protocolized acute burn care, enhanced wound coverage, improved resuscitation, and suitable infection control, severe burns still affect nearly every organ system resulting in significant morbidity and mortality [1-6]. A significant increase in circulating

\footnotetext{
* Correspondence: saeid.amininik@utoronto.ca; marc.jeschke@sunnybrook.ca ${ }^{1}$ Sunnybrook Research Institute, 2075 Bayview Ave., Rm. D704, Toronto, ON M4N 3M5, Canada

Full list of author information is available at the end of the article
}

catecholamines, glucocorticoids, glucagon, and dopamine secretion is thought to initiate the cascade of events leading to this hypermetabolic response [7-9]. A hallmark of the hypermetabolic response is significant muscle wasting, weakness, and debilitation, which persists for the duration of the hypermetabolic response [10,11]. This muscle wasting occurs in muscles distal to the burn site and is due to proteolysis to provide proteins and amino acids for the hugely increased metabolic demands. While this process is per se needed, the ensuing catabolism and associated weakness complicate and delays recovery. Several groups are trying to identify novel treatment approaches to mitigate this catabolism and hypothesize that a reduced catabolic response would improve outcomes after burn. 
Although investigations to date have identified probable leads and developed useful strategies to manage inflammation and muscle cachexia, no satisfactory drugs are yet available to curtail these conditions.

Metformin is an anti-diabetic agent that is recommended as a first-line oral therapy for type 2 diabetes (T2D) [12]. Metformin manages hyperglycemia by decreasing hepatic glucose production through the inhibition of mitochondrial respiratory-chain complex 1 [13]. With regard to skeletal muscle, metformin increases glucose uptake through upregulation of the glucose transporter type 4 (GLUT4) [14-17]. In addition to these effects, metformin also activates the cellular energy sensor, AMP-activated protein kinase (AMPK) [17], which has a wide range of effects throughout the body in numerous organs which will be discussed in more detail later. The activation of AMPK leads to the inhibition of hepatic gluconeogenesis and peripheral glucose uptake in the skeletal muscle [17]. This activation of AMPK leads to the translocation of GLUT4 and increased glucose uptake and glycolysis within the skeletal muscle. Under conditions of severe burn injury, adenylate cyclase converts ATP to AMP, reducing levels of ATP and increasing AMP $[18,19]$. This conversion reaches its highest peak at $72 \mathrm{~h}$ post-burn injury and results in activation of AMPK and phosphorylation of mTOR which initiates autophagy pathways $[18,19]$.

The therapeutic potential of metformin is not just limited to its ability to manage hyperglycemia and diabetes. Recently, metformin has been shown to effectively treat several diseases including cancer [20-22], cardiovascular diseases [23], and brain trauma [24, 25]. Moreover, there is evidence to suggest that metformin's pleiotropic effects delay the aging process [26, 27]. Several studies have shown that metformin can rescue muscle wasting in response to cardiovascular injury or skeletal muscle injury caused by cardiotoxin [28-31]. Recently, metformin has been found to promote the differentiation of human and mouse neural stem cells in culture. Moreover, after brain injury, metformin treatment increases the proliferation of endogenous neural stem cells, increases their total number of neural stem cells, and improves sensory-motor function after brain injury in mice [24]. Considering metformin's beneficial effects on neural stem cells in the context of injury, it is plausible that metformin has a similarly beneficial effect on muscle progenitor cells.

Skeletal muscle regeneration is dependent on contribution from muscle resident stem cells, named satellite cells. Satellite cells are marked by the paired-box transcription factor 7 (Pax7). Satellite cells are essential for skeletal muscle regeneration following injury [32] and for muscle hypertrophy and homeostasis [33-35]. Recent reports have illustrated a reduction in satellite cell numbers and an increase in myonuclear apoptosis post-burn injury in both humans and mice [36, 37]. Dysregulation of satellite cells may impair their ability to repair skeletal muscle after thermal injury. Indeed, depletion of satellite cells worsens muscle catabolism in mice after scald-burn injury [38] illustrating the importance of satellite cells in the recovery of lean muscle mass. Metformin is a drug that could potentially target satellite cells to prevent their dysregulation after thermal injury leading to less erosion of muscle mass. Gore et al. demonstrated that metformin treatment increases protein synthesis in severe burn patients [39]. One explanation for this increase in protein synthesis may be metformin increasing the proliferation of muscle progenitor cells (satellite cells). These findings suggest the diverse effects of metformin may extend into treating burn patients and improving their outcomes. Insulin resistance and muscle wasting are chronic complications of burn trauma. Metformin targets both insulin resistance and muscle wasting and is economically beneficial and easily administered orally. These advantages make it an attractive alternative for the long-term treatment of burn patients.

To date, the effects of metformin on muscle proteolysis and structure are essentially unknown. Using a 30\% total burn surface area (TBSA) murine burn model, we examined the effect of metformin treatment on mitigating burn-induced muscle wasting. We hypothesized that metformin treatment would (1) increase the proliferation of satellite cells after severe burn injury in the gastrocnemius muscle distal from the burn site and (2) attenuate muscle wasting after severe burn injury.

\section{Materials and methods}

\section{Mice}

All mice used were male, 8 weeks old, and C57BL/6. Mice were randomly divided into the following groups: sham, burn, and burn + metformin treatment $(n=18$ per group). Within each group, mice were subdivided into groups sacrificed at three different time points: 2 days, 7 days, and 14 days post-thermal injury ( $n=6$ per group). The animal experiments were performed in accordance with the guidelines and regulations set forth by the Sunnybrook Research Institute and Sunnybrook Health Sciences Animal Policy and Welfare Committee of the University of Toronto, Ontario Canada. The Sunnybrook animal care committee approved all animal experiments (approval \#15-503(M-1)) under the auspices of the Canadian Council on Animal Care.

\section{Burn}

Animals were anesthetized with isoflurane and received an intraperitoneal (IP) buprenorphine injection $(0.1 \mathrm{mg} / \mathrm{kg})$. The dorsum of the animal was shaved, and lactated Ringer's solution was subcutaneously injected along the spine. The mice were placed in a mold that exposes the dorsum to a 
pre-determined TBSA. A 30\% TBSA full-thickness scald injury was induced by exposing the dorsum of the animal to water pre-heated to $98^{\circ} \mathrm{C}$ for $10 \mathrm{~s}$ and the ventrum for 2 s. Following the burn, the animals were placed in separate cages. Sham animals were anesthetized and received buprenorphine injection but did not receive a thermal injury.

\section{Metformin treatment and cell proliferation analysis}

Each animal in the metformin group was injected intraperitoneally with $100 \mathrm{mg} / \mathrm{kg}$ of metformin hydrochloride (Sigma-Aldrich) dissolved in $1 \times$ PBS. Injections began 24 $\mathrm{h}$ after burn injury and continued every day at the same time until endpoint. To analyze cell proliferation in the skeletal muscle, we injected animals with 5-Bromo-2'deoxyuridine (BrdU) (Sigma) 24 h prior to harvest. Each animal received an intraperitoneal injection with $250 \mu \mathrm{L}$ of $2 \mathrm{mg} / \mathrm{ml} \mathrm{BrdU}$.

\section{Muscle harvest and dry/wet muscle ratio}

We dissected the gastrocnemius muscle from mice 2, 7, and 14 days after exposure to cutaneous thermal injury for histological and protein analysis. Whole gastrocnemius muscle was also weighed at the time of harvest to obtain the wet muscle weight. The dry muscle weight was obtained by dehydrating the whole gastrocnemius muscle for 5 days at $50^{\circ} \mathrm{C}$. The dry muscle weight was weighed. The dry muscle weight was divided by the wet muscle weight to obtain the dry/wet muscle ratio. For histology, muscle samples were tied to a support prior to excision to prevent contraction. Samples were either snap frozen in liquid nitrogen-cooled isopentane or fixed in $10 \%$ neutral buffered formalin for $24 \mathrm{~h}$.

\section{Hematoxylin and eosin staining (H\&E)}

Frozen sections were allowed to dry for $5 \mathrm{~min}$ at room temperature. Sections were then stained with Mayer's hematoxylin (Sigma-Aldrich) for $10 \mathrm{~min}$ and rinsed in running tap water. Sections were then dipped in $0.5 \%$ eosin 12 times and dipped in distilled water until eosin stops streaking. Sections were then dehydrated in various ethanol solutions and xylene. Finally, slides were mounted and cover slipped with xylene-based aqueous mounting media (SHUR/Mount ${ }^{\mathrm{Tm}}$ ).

\section{Myofiber cross-sectional area analysis}

Representative images of the gastrocnemius muscle sections were captured at $\times 20$ magnification. The crosssectional area of individual myofiber was obtained through Image $^{\circ}$ software. For each animal, the cross-sectional areas of approximately 500 myofibers were counted blindly in five images (field-of-views) and subsequently the average cross-sectional area was determined. We did not differentiate between type I and type II fibers when measuring muscle cross-sectional area.

\section{Western blot}

The gastrocnemius muscle was harvested, and protein was isolated from tissue lysates using RIPA lysis buffer. Protein concentrations were then measured using bicinchoninic acid (BCA) assay as previously reported [40]. Briefly, $30 \mathrm{mg}$ of each protein sample was separated by SDSpolyacrylamide gel electrophoresis, transferred to a nitrocellulose membrane, blocked with $5 \%$ milk in tris-buffered saline $/ 0.1 \%$ Tween 20 , and hybridized with the following primary antibodies: anti-Pax7 (1:500, DHSB), antiAMPK $\alpha$ (1:1000, Cell Signaling), anti-Phospho-AMPK $\alpha$ (Thr172) (1:1000, Cell Signaling), and GAPDH (1:5000, Cell Signaling). The membranes were then incubated with anti-rabbit or anti-mouse horseradish peroxidase (HRP)conjugated secondary antibody (1:2500, Santa Cruz). Detection of the signal was accomplished using western HRP chemiluminescence (ECL) reagents (Bio-Rad Laboratories), and imaging of the blots was performed using ChemiDoc $^{\mathrm{ma}}$ MP System (Bio-Rad). To analyze the blots, Image Lab ${ }^{\text {Tw }}$ Software (Bio-Rad) was used to quantify band intensity and calculate the absorbance ratio of the target protein to the loading control, GAPDH.

\section{Immunohistochemistry}

Gastrocnemius muscle samples for histological analysis were collected and fixed in $10 \%$ formalin for $24 \mathrm{~h}$ and transferred to $70 \%$ ethanol. Samples were then embedded in paraffin and sectioned at $5 \mu \mathrm{m}$ across the transverse plane. Paraffin-embedded slides were heated at $60^{\circ} \mathrm{C}$ for $30 \mathrm{~min}$ and deparaffinized with citrosol and rehydrated through a series of decreasing alcohol concentrations. Antigen decloaker solution (Biocare Medical) was preheated in an antigen decloaking chamber at $70{ }^{\circ} \mathrm{C}$ for $20 \mathrm{~min}$ before slides were added. The slides were then heated at $100^{\circ} \mathrm{C}$ in the antigen decloaking solution for $4 \mathrm{~min}$, cooled to $60^{\circ} \mathrm{C}$, and washed with tap water. After blocking endogenous peroxidase activity with $3 \% \mathrm{H}_{2} \mathrm{O}_{2}$ for $10 \mathrm{~min}$, sections were incubated with the following primary antibodies: anti-Pax7 (1:100, DHSB), anti-MPO (1:200, Abcam), and anti-NF-kB p65 (1:200, Cell Signaling). Slides were washed with washing buffer $(0.05 \mathrm{M}$ Tris- $\mathrm{HCl}, 0.15 \mathrm{M} \mathrm{NaCl}$, and $0.05 \%$ Tween 20 in double distilled water). Sections were then incubated in MACH3 probe (Biocare Medical) for $15 \mathrm{~min}$ and washed, and MACH3 horseradish peroxidase polymer detection was added for $15 \mathrm{~min}$. After washing again, betazoid diaminobenzidine (DAB) chromogen kits (Biocare Medical) were mixed and incubated for $10 \mathrm{~min}$, or until the brown color was observed. Slides were rinsed in running tap water, stained with hematoxylin for $30 \mathrm{~s}$, washed, and differentiated in 1.5\% acid alcohol briefly. Slides were then placed in $0.1 \%$ sodium bicarbonate for $10 \mathrm{~s}$ and dehydrated in citrosol and alcohol solutions. Lastly, slides were mounted and cover slipped 
with xylene-based aqueous mounting media (SHUR/ Mount $\left.{ }^{\mathrm{TN}}\right)$.

For quantification, five different fields were randomly chosen for each sample. The sections were imaged via an optical microscope (Leica Microsystems) with $\times 20$ and $\times 40$ objective lenses. The percentage of positive cells for each target was determined by dividing the number of positive cells by the total number of nuclei in each histological field. The average ratio for each subject was considered. Negative controls without primary antibody but with DAB staining were prepared to confirm the staining observed.

\section{Immunofluorescence}

Frozen muscle samples were embedded in OCT and frozen in liquid nitrogen-cooled isopentane. The samples were cut perpendicularly via a cryostat $(10-\mu \mathrm{m}$ thickness). Sections were allowed to cool for $5 \mathrm{~min}$ at room temperature and subsequently fixed in $4 \%$ paraformaldehyde (PFA) for $5 \mathrm{~min}$. Sections were washed with PBS and incubated with glycine solution to quench the PFA signal. For BrdU staining, sections were incubated in 1.5 $\mathrm{M} \mathrm{HCl}$ for $30 \mathrm{~min}$ at $37^{\circ} \mathrm{C}$ and subsequently neutralized in $0.1 \mathrm{M}$ borate buffer solutions for $5 \mathrm{~min}$. After washing, sections were permeabilized in $0.25 \%$ Triton- $X$ for $10 \mathrm{~min}$ and washed again. Sections were then incubated in blocking buffer (5\% normal goat serum, 2\% BSA, and mouseon-mouse blocking reagent diluted PBS) for $1 \mathrm{~h}$ at room temperature. Sections were rinsed in PBS and incubated in primary antibody overnight at $4{ }^{\circ} \mathrm{C}$ overnight. The following antibodies were used: anti-Pax7 (1:100, mouse, DHSB), anti-BrdU (1:250, rat, Abcam), and Laminin (1: 200, rabbit, Abcam). Sections were rinsed with PBS and incubated in secondary antibody solution diluted in blocking buffer: goat anti-rabbit Alexa Fluor 488 (1:1000), goat anti-mouse IgG1 Alexa Fluor 546 (1:1000), or goat antimouse IgG1 Alexa Fluor 488 (1:1000). Sections were rinsed and mounted with fluorescent mounting media containing DAPI (Vector Laboratories). Samples were imaged with a Zeiss Apotome fluorescent microscope.

\section{Oil Red $\mathrm{O}$ staining}

The gastrocnemius muscle was harvested, snap frozen in liquid nitrogen-cooled isopentane, and embedded in OCT. Using a cryostat, the muscle was cut perpendicularly (thickness $10 \mu \mathrm{m}$ ) and added onto the slide. Slides were fixed in formalin and briefly washed with running tap water. Sections were then rinsed in $60 \%$ isopropanol and stained with freshly prepared Oil Red O working solution for $15 \mathrm{~min}$. After another rinse with $60 \%$ isopropanol, nuclei were lightly stained with hematoxylin (Sigma-Aldrich) for $30 \mathrm{~s}$ and rinsed with distilled water. Slides were mounted and cover slipped with xylenebased aqueous mounting media (SHUR/Mount ${ }^{\mathrm{Tm}}$ ).

\section{Statistical analysis}

Statistical analysis was performed using one-way ANOVA. Data are represented as mean $\pm \operatorname{SEM}(n=6) . p<0.05$ were taken as statistically significant.

\section{Results}

\section{Metformin treatment attenuates muscle wasting in mice}

To assess whether metformin attenuates muscle catabolism after severe burn injury, we examined animal weights, the dry/wet muscle ratio, and the cross-sectional muscle area. As expected, animal weights in the burn groups decreased significantly at 7 days, and this was sustained until 14 days post-thermal injury (Fig. 1a), $p<0.05$. This corresponded to a $5 \%$ decrease in weight. In contrast, with metformin treatment, there was only a $2.5 \%$ decrease in weights, a difference that is significant compared to the burn groups (Fig. 1a), $p<0.05$. This indicates that metformin attenuates muscle catabolism post-thermal injury. Lastly, the change in muscle weight relative to body weight (muscle weight/ body weight) can be seen in Additional file 1 .

To examine changes in muscle histology, we performed hematoxylin and eosin (H\&E) staining. There was a significant reduction in myofiber cross-sectional area at these 7 days in the burn group (Fig. 1c, d), $p<0.05$. Several studies have shown that metformin can rescue muscle wasting in response to cardiovascular injury or skeletal muscle injury caused by cardiotoxin [28-31]. Indeed, in our burn-induced muscle wasting model, metformin treatment attenuated muscle wasting at 7 days post-thermal injury (Fig. 1c, d), $p<0.05$. There was no significant difference in dry/wet muscle ratio and muscle cross-sectional between the metformin-treated group and the sham group at 7 days post-thermal injury (Fig. 1b). These results suggest that metformin mitigates muscle wasting in severely burned mice.

\section{Metformin does not affect myofiber size at $\mathbf{1 4}$ days}

At 14 days post-thermal injury, we observed no differences in the dry/wet muscle ratio or the muscle cross-sectional area between sham, burn, and metformin groups (Fig. 2a, b). We hypothesize that by day 14 post-thermal injury, the mice have likely recovered in terms of their lean muscle mass regardless of metformin treatment. This might be expected due to the reduced morbidity observed in mice as a result of accelerated healing time [41] and differences in immune function [42] compared to humans. Moreover, as opposed to humans, mice are highly mobile after burn injury which may accelerate recovery of muscle mass. Metformin seems to exert its effect on the skeletal muscle and myofiber size early in the burn response of mice.

\section{Metformin treatment reduces fat infiltration in the skeletal muscle}

After burn injury, there is massive lipolysis and release of free fatty acids that lead to fat infiltration 
A

C
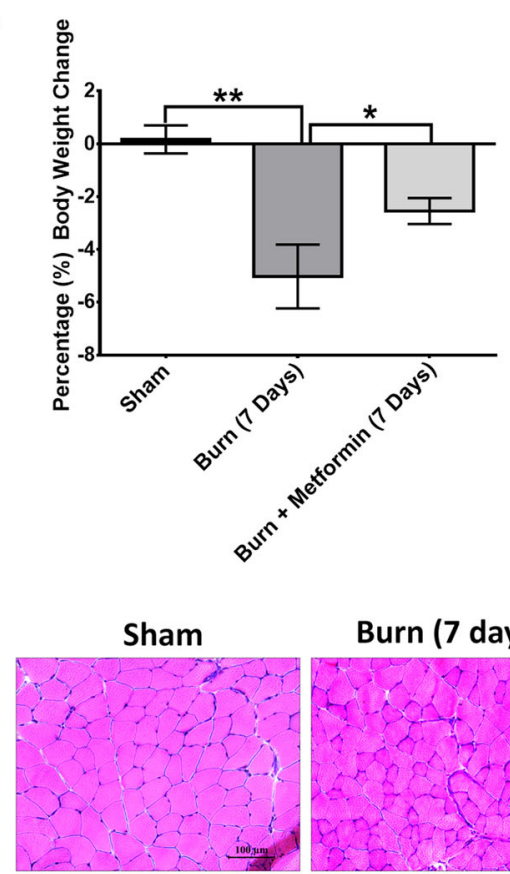

Burn (7 days)

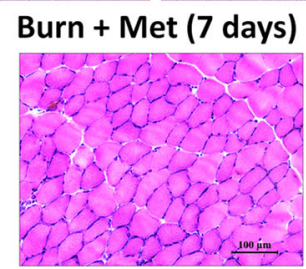

B

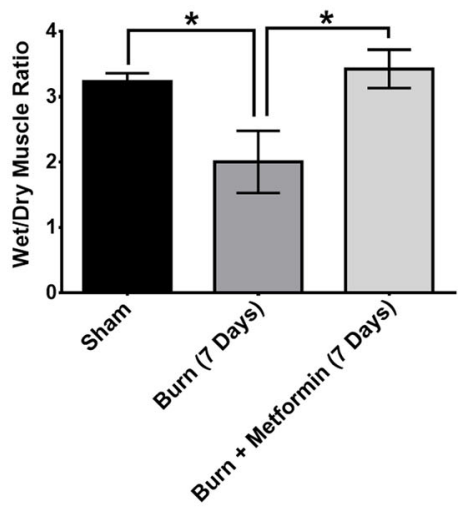

D

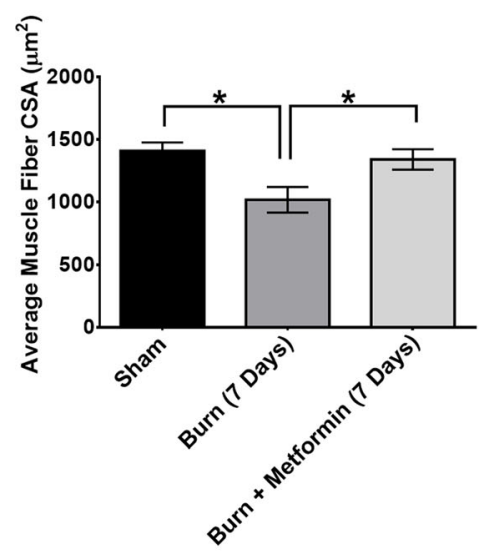

Fig. 1 Metformin treatment attenuates muscle wasting in severely burned mice. a The percentage (\%) body weight change in sham, burn, and burn + metformin at 7 days post-burn in mice. b Gastrocnemius muscle mass expressed as a dry/wet muscle ratio in sham, burn, and burn + metformin at 7 days post-burn in mice. c Representative images of hematoxylin and eosin staining of the gastrocnemius muscle from sham, burn, and burn + metformin mice at 2 and 7 days post-burn injury. Myofibers are significantly smaller at 7 days post-burn injury. Metformin treatment restores myofiber size. Images were taken at $\times 20$ magnification. $\mathbf{d}$ Quantification of muscle cross-sectional area $\left(\mu m^{2}\right)$ in sham, burn, and burn + metformin at 2 and 7 days post-burn injury

in several organs, including the liver and skeletal muscle [43]. Intramuscular fat accumulation in the skeletal muscle is linked with decreased muscle strength, reduced insulin sensitivity, and increased mortality [44]. Metformin has numerous systemic effects, one of which is reducing fat infiltration in the liver [45-47] and kidney [48]. Therefore, metformin may have indirect effects on the skeletal muscle such as reducing fat infiltration that facilitates recovery of lean muscle mass. To examine fat infiltration in the muscle, we performed Oil Red $\mathrm{O}$ staining to visualize intramuscular lipid droplets. There was fat infiltration at 7 days post-thermal injury in the burn group when compared with sham and metformin animals (Fig. 3). With metformin treatment, we observed less fat infiltration compared to the burn group (Fig. 3). The extent of Oil Red O staining in the metformin group was comparable to the sham group (Fig. 3).

\section{Metformin treatment activates AMPKa in the skeletal} muscle after severe burn injury

To evaluate pathway activation of metformin in our burn model, we performed western blotting for AMPK $\alpha$ and the activated version of AMPK, phospho-AMPK $\alpha$ (Thr172). We observed no significant difference in the protein level of AMPK $\alpha$ between the sham, burn-, and metformin-treated groups at 7 days (Fig. 4a, b). However, there was a significant increase in the protein level of phospho-AMPK $\alpha$ in the metformin treatment group at 7 days post-thermal injury (Fig. $4 \mathrm{a}, \mathrm{b}$ ), $p<0.05$. We did not observe an increase in phospho-AMPK $\alpha$ in the burn group. This might be because 7 days post-burn is too late of a time point to examine as activation of AMPK occurs during the acute phase of the burn response (i.e., $72 \mathrm{~h}$ or less) $[18,19]$. Nevertheless, these results confirm that metformin-activated AMPK and AMPK $\alpha$ phosphorylation may be the mediator by which metformin alleviated muscle catabolism. Studies suggest that chronic activation 
A

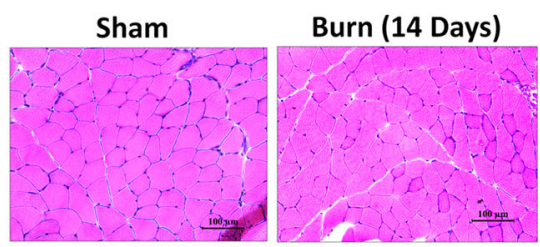

Burn + Met (14 Days)

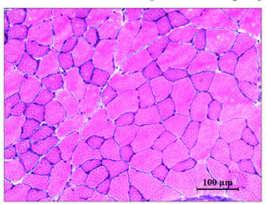

B

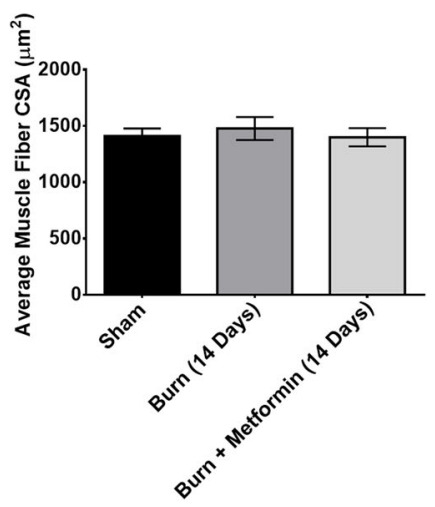

Fig. 2 Metformin has no effect on myofiber size at 14 days. a Representative images of hematoxylin and eosin staining of the gastrocnemius muscle from sham, burn, and burn + metformin mice at 14 days post-burn injury. Images were taken at $\times 20$ magnification. $\mathbf{b}$ Quantification of muscle cross-sectional area $\left(\mu \mathrm{m}^{2}\right)$ in sham, burn, and burn + metformin at 14 days post-burn injury

of AMPK $\alpha$ in the skeletal muscle increases the expression of muscle hexokinase and glucose transporter 4 (GLUT4), which mimics the effects of exercise training in muscle [49].

Metformin treatment increases the number of Pax7+ cells and protein level at 7 days in the skeletal muscle after severe burn injury

The importance of $\mathrm{AMPK} \alpha$ activation in attenuating burn-induced muscle wasting is further supported by the fact that AMPK $\alpha$ activation in satellite cells is essential for muscle regeneration [50]. To examine whether metformin treatment influences satellite cell activity after severe burn injury, we performed immunohistochemistry and western blotting for Pax7, a transcription factor expressed by quiescent and proliferating satellite cells. Protein expression of Pax7 in the burn group was significantly reduced in the muscle at 7 days post-thermal injury when compared with sham and metformin groups, which is in line with our previous report (Fig. 5a, b) [37]. Interestingly, metformin significantly increased protein expression of Pax7 compared to sham and burn groups (Fig. 5a, b), $p<$ 0.05. Immunohistochemistry revealed a significant reduction in the number of Pax7-positive nuclei in the burn group compared to sham and metformin-treated groups at 7 days post-thermal injury (Fig. 5c, d), $p<0.05$. Metformin treatment significantly increased the number of Pax7positive nuclei compared to sham and burn groups (Fig. $5 c, d), p<0.05$. This increase in the number of satellite cells may have contributed to the rescue of muscle

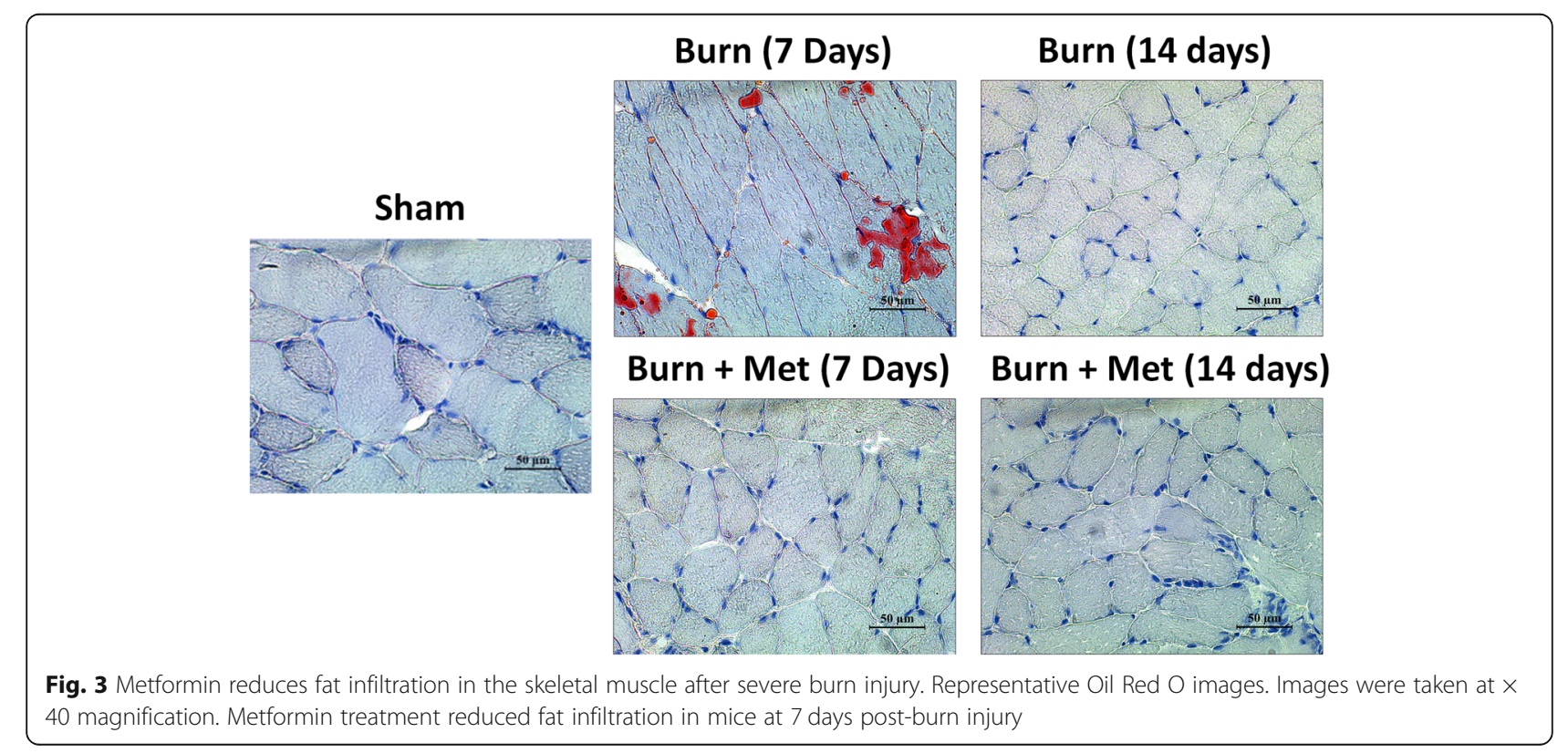


A

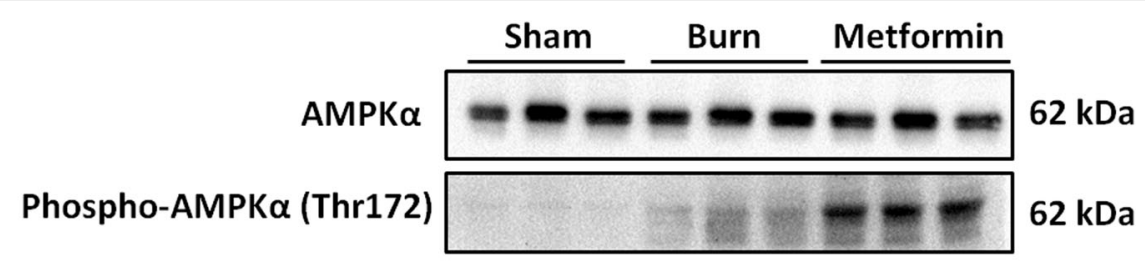

B

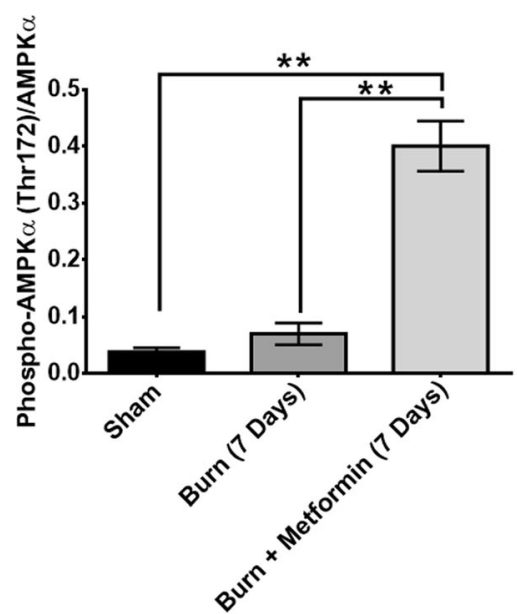

Fig. 4 Metformin treatment activates AMPKa in the skeletal muscle after severe burn injury. a Representative western blot for AMPKa and phospho-AMPKa (Thr172). b Quantification of phospho-AMPKa (Thr172) protein level normalized to AMPKa

wasting observed in metformin-treated mice. Another possibility is that metformin enhanced anabolism of the skeletal muscle at an earlier time point post-burn resulting in the recovery of Pax7+ cells earlier. A greater pool of satellite cells (muscle progenitors) increases the regenerative capacity of the skeletal muscle to build new myofibers.

\section{Metformin treatment increases the proliferation of satellite cells in the skeletal muscle}

To further assess whether metformin affects satellite cells or the changes in satellite cells are secondary to the effect of metformin on muscles after severe burn injury, we investigated metformin's effect on the proliferation of these stem cells. The increase in Pax7-positive cells observed in the metformin group at 7 days may be due to increased proliferation caused by metformin. Recently, metformin has been shown to increase the absolute number of neural precursor cells in mice and increase their proliferation in response to brain injury [24, 25]. To determine whether metformin has a similar effect in satellite cells, we performed immunofluorescence double staining for Pax7 and BrdU to identify muscle progenitors that were proliferating. Animals were injected with BrdU $24 \mathrm{~h}$ prior to sacrifice to label proliferating myonuclei. Quantification of the proportion of Pax7/BrdU-positive cells revealed a significant increase in proliferating Pax7positive cells as well as a total number of Pax7-positive cells (Fig. 6a, b), $p<0.05$ (Additional file 2). Fifteen percent of Pax7-positive cells were positive for BrdU in the metformin group indicating a proliferation rate of $15 \%$ after burn injury (Fig. 6b). Collectively, these data suggest that metformin increases the proliferation and the total number of muscle progenitor cells in the skeletal muscle after severe burn injury. Metformin's mitigation of muscle wasting after burn injury may in part be due to its beneficial effects on muscle progenitors and the regenerative capacity of the skeletal muscle.

\section{Metformin treatment does not attenuate inflammation in the skeletal muscle of severely burned mice}

We have recently shown that the reduction in satellite cells post-burn injury is tightly associated with an inflammatory cascade. Metformin has anti-inflammatory properties [51]. Studies have suggested that metformin suppresses inflammation in diabetes and intestinal inflammation by inhibiting the activity of NF- $\mathrm{kB}$ via AMPK-independent and AMPK-dependent processes [52-55]. Metformin's effect on satellite cells may in part be due to its anti-inflammatory effects. To investigate metformin's effect on inflammation after severe burn injury, we performed immunohistochemistry and western blotting for NF-kB p65. This protein complex has been implicated in causing muscle wasting in several different diseases [56, 57]. Activation of NF- $\mathrm{kB}$ results in the transcription of muscle-specific ubiquitin ligases such as MurF1 that cause 


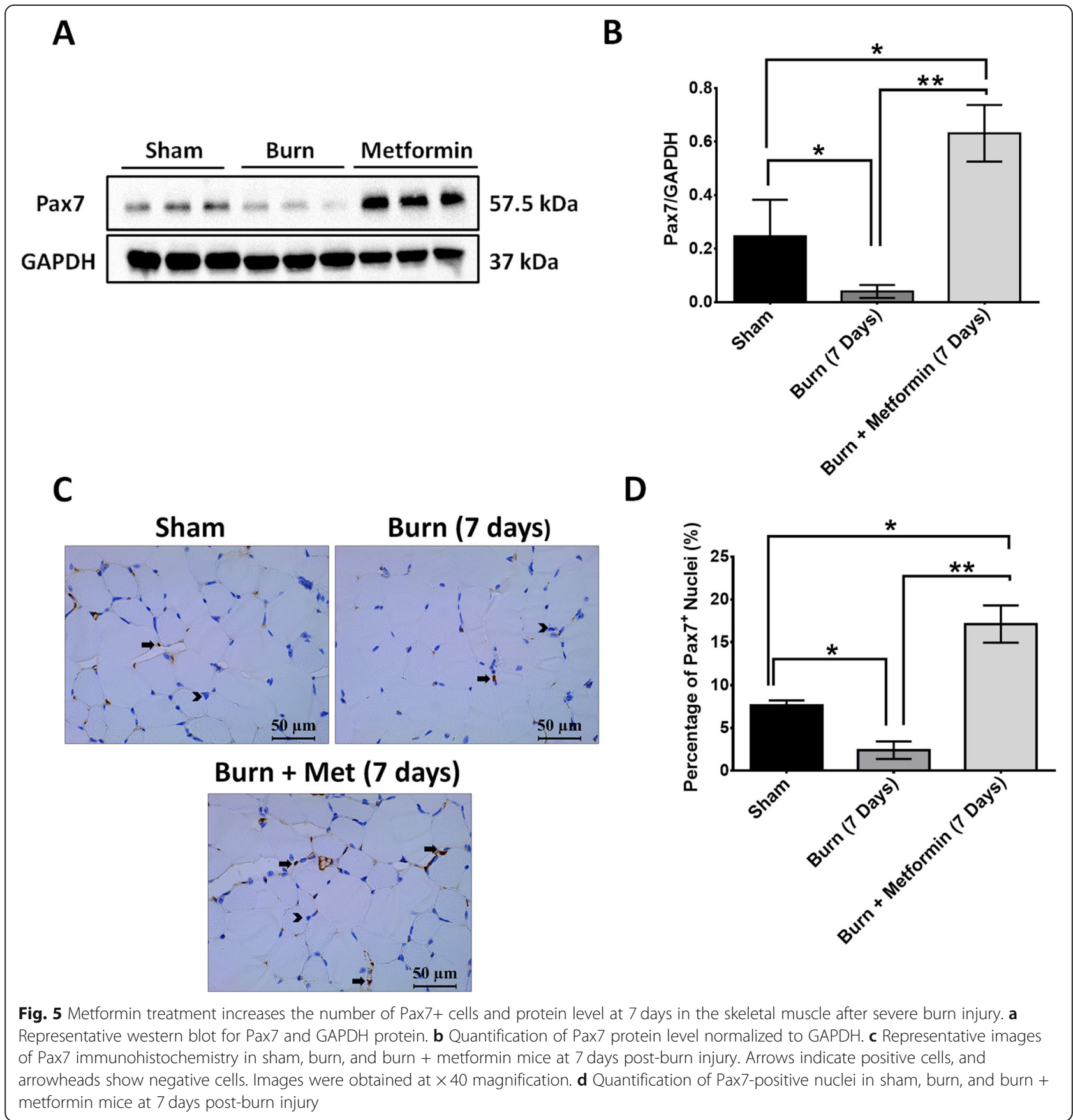

protein degradation $[58,59]$. Furthermore, NF-kB p65 has been shown to be elevated in the skeletal muscle of mice [37] and in the serum of burn patients [60]. We observed a significant increase in NF- $\mathrm{KB}$ p65 protein level and the number of NF-kB p65-positive myonuclei after severe burn injury (Fig. 7), $p<0.05$. Unlike previous studies, metformin treatment did not attenuate NF- $\mathrm{kB}$ p65 activity in this model. It is possible that metformin's mitigation of muscle atrophy may be attributed to its effect on satellite cell proliferation rather than its anti-inflammatory properties. Further studies are needed to confirm this effect.

\section{Discussion}

Numerous studies have illustrated the protective effect of metformin in mitigating skeletal muscle damage [28$31,61,62]$ and its modulation of stem cell function in the context of injury [24]. Given that metformin is a metabolic drug that can potentially enhance muscle regeneration and stem cell function, we investigated the effect of metformin on the skeletal muscle in response to burn injury. Here, we show that metformin treatment attenuates muscle wasting in response to burn-induced skeletal muscle wasting. Metformin treatment increased 


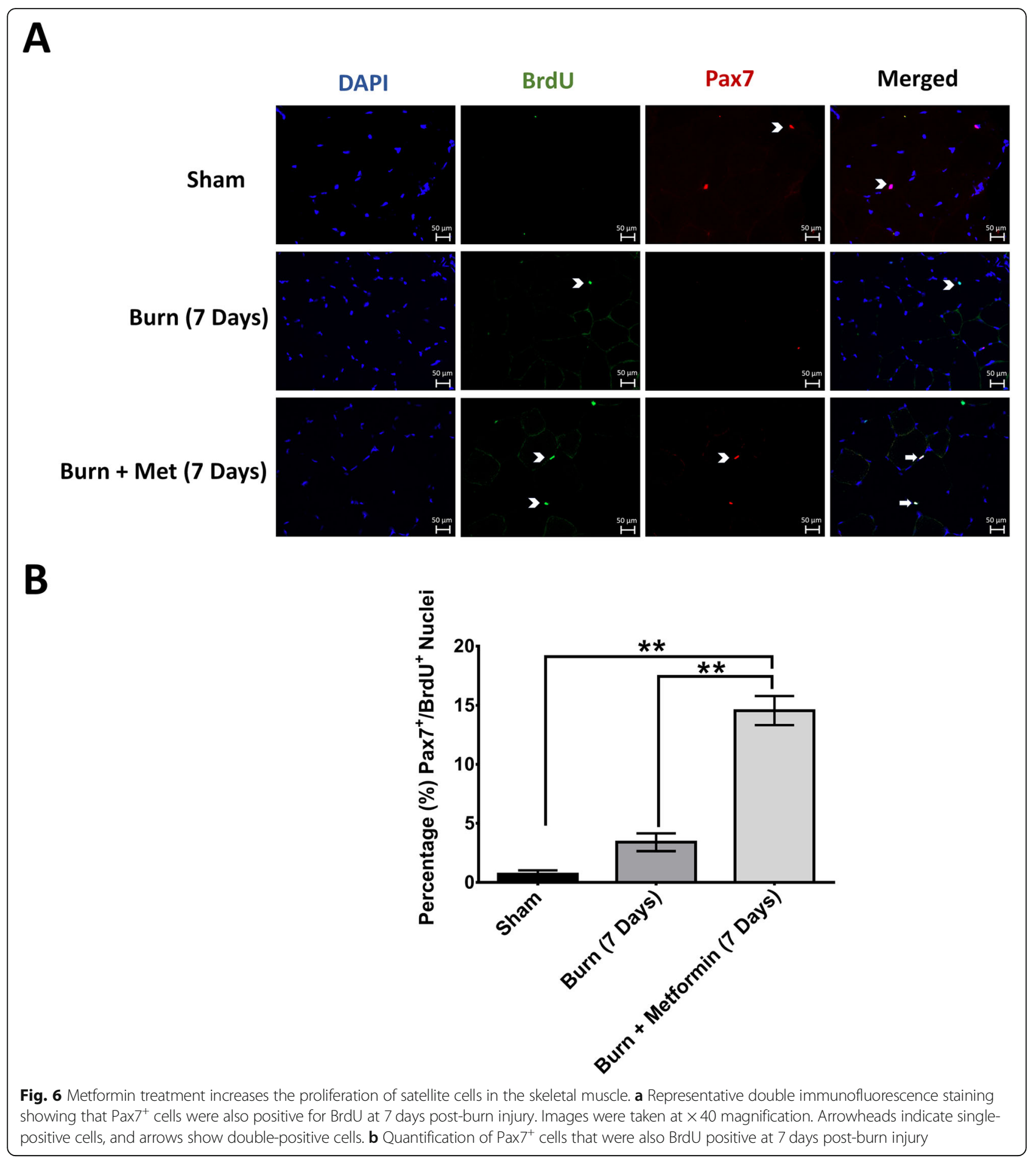

gastrocnemius muscle weight and muscle cross-sectional area when compared with the non-treated burn group (Fig. 1b), $p<0.05$. There was no significant difference between the sham and metformin groups at 7 days indicating a recovery of muscle mass with metformin treatment (Fig. 1b). This rescuing of muscle atrophy is consistent with previous findings showing that metformin rescues muscle wasting in other injury models. Metformin's attenuation of muscle atrophy after burn injury was also supported by our measurements of the myofiber cross-sectional area (Fig. 1c, d).

To characterize metformin's mechanisms in mitigating burn-induced muscle wasting, and with the consideration that our latest report revealed temporal changes in Pax7- 


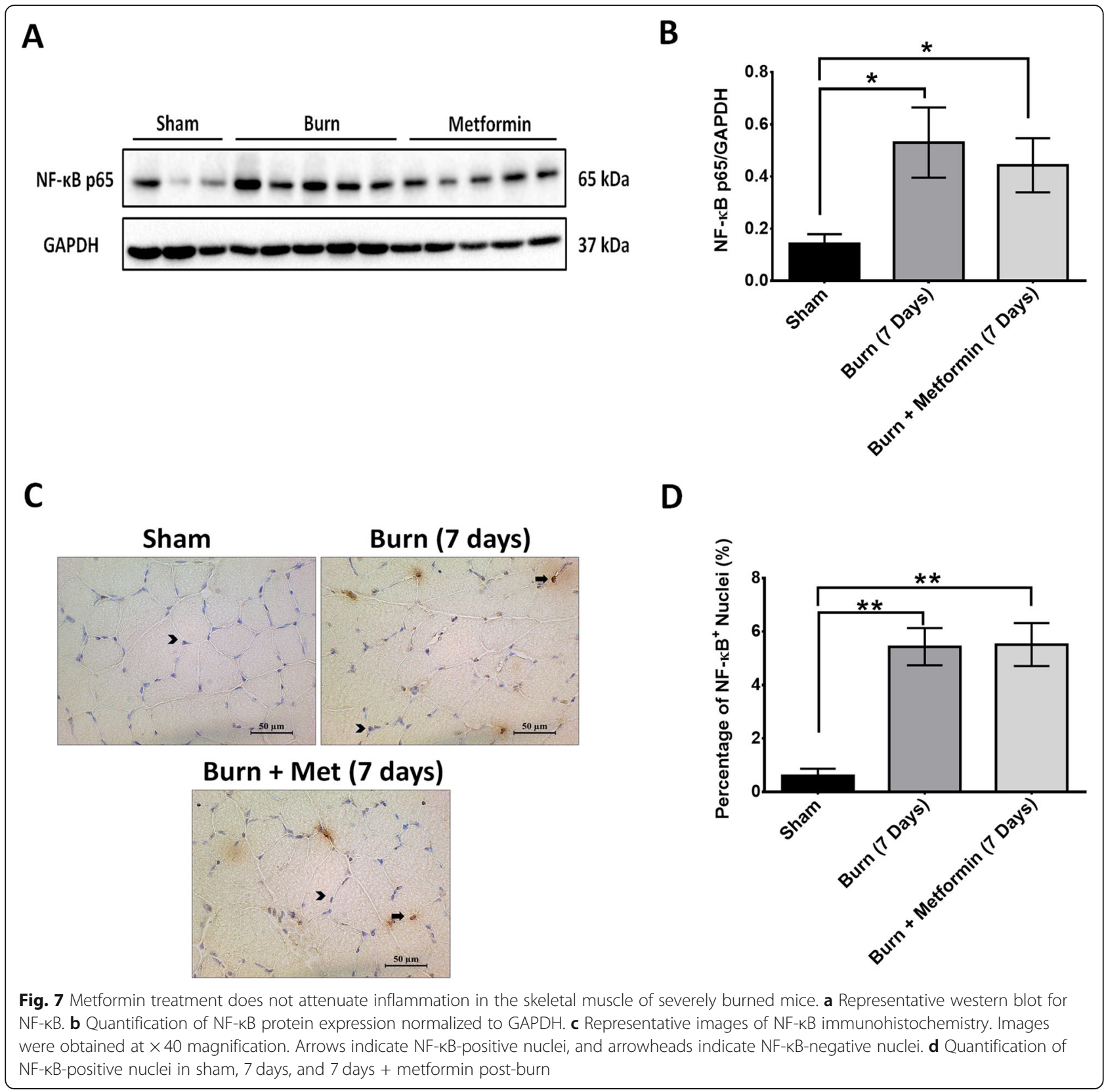

positive muscle progenitor cells post-thermal injury [37], we examined how satellite cells responded to metformin treatment. Metformin treatment significantly increased the number of muscle progenitors $\left(\mathrm{Pax}^{+}\right)$and the protein level of Pax7 when compared with sham and burned animals (Fig. 5), $p<0.05$. Metformin treatment also significantly increased the proliferation of satellite cells (Fig. 6) as approximately $15 \%$ of Pax $7+$ cells were BrdU positive, $p<0.05$. In various models of muscle regeneration, a proliferation rate of $15-20 \%$ is reported after injury in the skeletal muscle [63-65]. Furthermore, as the severity of muscle injury increases (e.g., polytraumatic injury), the proliferation rate increases to $28 \%$ after 1 week post injury
[66]. Although the method of inducing muscle injury differs between these studies (e.g., cardiotoxin, cold lesion injury) and our model of burn-induced muscle wasting, they all present almost similar levels of muscle atrophy. As such, the similar proliferation rate araise in our study was expected. Mechanistically, metformin's metabolic effects might be the underlying mechanism for this proproliferatory effect of it. Activated satellite cells proliferate to expand their population and undergo myogenic differentiation into new myofibers [67]. Studies show that stem cells, including satellite cells, rely on glycolysis to provide energy and proliferate $[68,69]$. This is due to their deep location within the tissue that limits access to oxygen and 
protects against damage from reactive oxygen species [70-72]. Satellite cells have few mitochondria and relatively small cytoplasm; hence, they normally have low metabolic rates [73]. When activated, the metabolism of satellite cells rapidly elevates to provide the energy needed for proliferation and differentiation [73]. Like cancer cells, satellite cells rely on Warburg-like glycolysis as the primary source of energy and fast proliferation [50, 74]. As discussed, metformin's main effect on the skeletal muscle is to increase glucose uptake and glycolysis. Therefore, it is plausible that metformin treatment provides more energy to proliferating satellite cells thus enhancing their ability to regenerate and repair damaged myofibers after burn injury. Recently, Pavlidou et al. reported that metformin reduced the number of $\mathrm{Pax}^{+} / \mathrm{BrdU}^{+}$muscle progenitor cells and depleted skeletal muscle regeneration, a finding contrary to ours [75]. The differences between the two studies may be the nature of the injury. We used a burn model to induce muscle wasting rather than cardiotoxin to induce "muscle crush injury." The local recruitment of inflammatory cells in cardiotoxin injury is different from the systemic inflammatory reaction to burn injury. For example, local muscle injury is characterized by a local increase in neutrophil activity and release of TNF $\alpha$ by M1 macrophages which is sustained for up to 2 weeks after injury [76]. Moreover, local cardiotoxin injury specifically increases the expression of osteopontin (OPN), a regulator of muscle inflammation, an event $48 \mathrm{~h}$ after injury [77]. Burn injury on the other hand results in a systemic cascade of proinflammatory such as IL-6, TNF, IL-15, MCP-1, and GM-CSF [6]. These cytokines decrease significantly at 2 weeks when there is a switch to anti-inflammatory phenotype [6]. This key difference changes in the nature of the injury between the two studies and may change metformin's effect on the skeletal muscle. Another difference between the two studies may be the mobility of mice after cardiotoxin injury versus burn injury. Our lab has shown that after severe burn injury, mice are quite mobile [78]. Cardiotoxin injury, however, significantly reduces the mobility of mice post injury [79]. As a result, the differences in mobility will affect the dynamics of muscle proliferation and differentiation, and thus muscle recovery. Lastly, another study showed that metformin protects against cardiotoxin-induced degeneration [31] and metformin's effects may be context-dependent [80].

To confirm metformin activity within the skeletal muscle after treatment, we performed western blotting for AMPK. AMPK is a master regulator of metabolism which has an $\alpha$ catalytic subunit with two isoforms, $\alpha 1$ and $\alpha 2$ [81]. AMPK's overall function in the skeletal muscle is to respond to cellular energy deprivation by increasing the potential for ATP production, and AMPK is typically activated during exercise [81]. We observed a significant increase in the protein level of the active form of AMPK $\alpha$, phospho-AMPK $\alpha$, in the metformin group after severe burn injury (Fig. 4), $p<0.05$. This is consistent with the literature showing that metformin exerts its effects through the activation of AMPK in the liver and skeletal muscle [17]. The activation of AMPK in the skeletal muscle after burn injury has important implications in metformin's observed effect. Recently, AMPK has been shown to be a critical mediator of satellite cell activation and muscle regeneration [50]. In transgenic mice with satellite cell-specific AMPK $\alpha 1$ knockout, there is impairment of the activation and myogenic differentiation of satellite cells during muscle regeneration, thus sustaining muscle atrophy [50]. Researchers also illustrated that activation of AMPK is essential for the Warburg-like glycolysis of satellite cells during muscle regenerations [50]. Therefore, the activation of AMPK we observed in the metformin-treated burn group might be another underlying mechanism to the increase in satellite cell proliferation and attenuate of burn-induced muscle wasting observed (Fig. 4).

Metformin may have also indirectly influenced satellite cell activity through decreasing fat infiltration in the skeletal muscle. After severe burn injury, lipid metabolism is significantly altered resulting in extensive lipolysis [82]. Lipolysis is the breakdown of triacylglycerol into free fatty acids (FFA) and glycerol [82]. The release of free fatty acids contributes to post-burn morbidity and mortality by mediating insulin resistance and increasing fat infiltration in various organs, including the skeletal muscle [82]. Intramuscular fat infiltration is the accumulation of fat within the myofibers themselves [44]. This is because free fatty acids impair insulin-mediated glucose uptake $[83,84]$ and inhibit glucose transport activity [85]. Furthermore, fat infiltration in the muscle is associated with increased risk of fracture and frailty [86], inflammation [87], and functional deficits [44]. In this animal study, we show that metformin reduces fat infiltration in the skeletal muscle after severe burn injury (Fig. 3). Recent findings from our lab have also shown that metformin reduces fat infiltration in the liver and improves mitochondria bioenergetics. One way by which metformin may reduce fat infiltration is through the activation of AMPK [80]. One of AMPK's many effects is to inhibit the activity of Acetyl-CoA carboxylase (ACC), a key enzyme in the synthesis of fatty acids [80]. A reduction in ACC activity by metformin treatment may reduce fatty acid synthesis after burn injury leading to a reduction in circulating fatty acids and thus less fat accumulation in organs such as the skeletal muscle and liver. Perhaps this reduction in intramuscular fat infiltration reduces inflammation in the skeletal muscle, thus improving the function of satellite cells and reducing the extent of muscle wasting observed. 
Severe burn injury is associated with insulin resistance and hyperglycemia. Clinically, this is detrimental to patients because it is associated with worse outcomes due to increased infections, increased catabolism and hypermetabolism, and increased incidence of pneumonia. The gold standard to treat hyperglycemia is insulin. Insulin treatment achieves tight glucose control and reduces the morbidity of patients. While this is encouraging, there are limitations to insulin treatment. For example, insulin treatment is associated with a fourfold increased risk of hypoglycemia. This is important because patients that experience a hypoglycemic episode have a ninefold increased risk of mortality [82]. Thus, the use of insulin in intensive care units is limited. Alternatively, treating burn patients with an anti-diabetic drug that manages glucose levels with fewer factors than insulin is ideal. Metformin is a drug that can achieve tight glucose control without the added risk of hypoglycemia like insulin. Gore et al. investigated the effect of metformin on severely burn adults through a stable isotope infusion study [39]. One group received metformin treatment $(n=8)$ for 7 days while another received the placebo $(n=5)$ for the duration of the study [39]. In the metformin group, endogenous glucose production decreased by $50 \%$, and serum glucose levels were significantly lower compared to the placebo group [39]. Researchers found that the rate of protein breakdown was unaffected despite the reduction in glucose production and levels [39]. However, there was a net improvement in protein balance in the metformin group due to an elevation in protein synthesis levels [39]. A possible downside to using metformin is the potential for patients to experience lactic acidosis. A randomized phase II clinical trial by Jeschke et al. has demonstrated that metformin decreased glucose levels equally as well as insulin and was safe to use in burn patients. Furthermore, metformin treatment is not associated with lactic acidosis in burn patients [82]. A systemic review of 347 clinical trials found no evidence of fatal lactic acidosis [88].

There are a few limitations to our study which could be addressed in future studies. First, besides in vivo, we can explore the mechanisms of metformin further through in vitro studies. For instance, metformin's effect on satellite cells can be confirmed by isolating satellite cells from humans or mice and treating them with metformin. Based on our current study, we would expect metformin to increase proliferation of satellite cells in vitro. Moreover, treating satellite cells with metformin and dorsomorphin (a reversible and selective AMPK inhibitor) and examining their effects on proliferation is important. This will help determine whether metformin's effect on satellite cells is through AMPK or some other mechanisms independent of AMPK. Unfortunately, a challenge with in vitro studies is replicating the inflammatory niche unique to burn injury. Therefore, we believe that the value of in vivo studies is greater. Second, repeating our study with Pax7 reporter mice to trace the lineage of proliferation and differentiation after severe burn injury would be insightful [35]. Furthermore, developing mice that are deficient for AMPK in Pax7 reporter cells is important to uncover the mechanisms of metformin on satellite cells after burn injury. Last, using mice models may not accurately replicate postburn hypermetabolism observed in burn patients [78]. Larger animal models like porcine would be more reflective of humans [78].

\section{Conclusion}

In summary, our work shows that metformin mitigates burn-induced muscle wasting in vivo through enhancement of a myogenic phenotype by affecting Pax7positive skeletal muscle progenitor cells. The underlying mechanism might mainly rely on the activation of AMPK, modulation of muscle progenitor activity, or reduction of fat infiltration in the muscle. These findings, in conjunction with recent findings illustrating the safety and efficacy of metformin treatment in burn patients, support the notion that long-term treatment with metformin could have beneficial effects in attenuating hypermetabolism and muscle catabolism in burn patients. Future research should focus on the development of therapies that address burn-induced alterations in satellite cell activity to maximize the recovery of muscle mass in burn patients.

\section{Supplementary information}

Supplementary information accompanies this paper at https://doi.org/10. 1186/s13287-019-1480-x.

Additional file 1. Ratio of muscle weight to body weight in sham, burn, burn + metformin at 7 days post-burn-in mice.

Additional file 2. Quantification of $\mathrm{BrdU}^{+} / \mathrm{Pax}^{-}$cells in sham, burn, burn + metformin at 7 days post-burn-in mice. B) Quantification of $\mathrm{Pax}^{+} / \mathrm{BrdU}^{-}$cells in sham, burn, burn + metformin at 7 days post-burnin mice.

\section{Abbreviations}

ACC: Acetyl-COA carboxylase; AMPK: AMP-activated protein kinase; CSA: Crosssectional area; FFA: Free fatty acids; GLUT4: Glucose transporter type 4; Pax7: Paired-box transcription factor 7; TBSA: Total burn surface area; T2D: Type 2 diabetes

\section{Acknowledgements}

The authors would like to thank all members of the Jeschke Lab for their help and the Canadian Institutes of Health Research for funding this study.

\section{Authors' contributions}

YY contributed to the conception and design, collection of data, data analysis, and manuscript writing. AD contributed to the collection of data, data analysis, and manuscript writing. BB contributed to the collection of data, data analysis, and manuscript writing. MGJ and SAN contributed to the conception and design, provision of study material/grants, data analysis, manuscript writing, and final approval of the manuscript. All authors read and approved the final manuscript. 


\section{Funding}

This study was supported by the Canadian Institutes of Health Research \# 123336 and CFI Leader's Opportunity Fund Project \# 25407 NIH RO1 GM087285-01

\section{Availability of data and materials}

The data generated from this study are available from the corresponding author upon reasonable request.

\section{Ethics approval and consent to participate} Not applicable.

\section{Consent for publication}

Not applicable.

\section{Competing interests}

The authors declare that they have no competing interests.

\section{Author details}

'Sunnybrook Research Institute, 2075 Bayview Ave., Rm. D704, Toronto, ON M4N 3M5, Canada. 'Laboratory in Medicine and Pathobiology, University of Toronto, Toronto, Canada. ${ }^{3}$ Division of Plastic Surgery, Department of Surgery, University of Toronto, Toronto, Canada. ${ }^{4}$ Ross Tilley Burn Centre, Sunnybrook Health Sciences Centre, Toronto, Canada. ${ }^{5}$ Department of Immunology, University of Toronto, Toronto, Canada.

\section{Received: 5 July 2019 Revised: 14 October 2019}

Accepted: 31 October 2019 Published online: 08 January 2020

\section{References}

1. Herndon DN, Tompkins RG. Support of the metabolic response to burn injury. Lancet. 2004;363(9424):1895-902.

2. Kraft $R$, et al. Burn size and survival probability in paediatric patients in modern burn care: a prospective observational cohort study. Lancet. 2012; 379(9820):1013-21.

3. Williams FN, et al. The leading causes of death after burn injury in a single pediatric burn center. Crit Care. 2009:13(6):R183.

4. Jeschke MG, et al. Threshold age and burn size associated with poor outcomes in the elderly after burn injury. Burns. 2016;42(2):276-81.

5. Patsouris $D$, et al. Burn induces browning of the subcutaneous white adipose tissue in mice and humans. Cell Rep. 2015;13(8):1538-44.

6. Jeschke $M G$, et al. Pathophysiologic response to burns in the elderly. EBioMedicine. 2015;2(10):1536-48.

7. Williams FN, Herndon DN, Jeschke MG. The hypermetabolic response to burn injury and interventions to modify this response. Clin Plast Surg. 2009; 36(4):583-96.

8. Mlcak RP, et al. The influence of age and gender on resting energy expenditure in severely burned children. Ann Surg. 2006:244(1):121-30.

9. Hart DW, et al. Persistence of muscle catabolism after severe burn. Surgery. 2000;128(2):312-9.

10. Pereira $C$, et al. Post burn muscle wasting and the effects of treatments. Int J Biochem Cell Biol. 2005;37(10):1948-61.

11. Jeschke MG, et al. Long-term persistance of the pathophysiologic response to severe burn injury. PLoS One. 2011;6(7):e21245.

12. Nathan DM, et al. Medical management of hyperglycaemia in type 2 diabetes mellitus: a consensus algorithm for the initiation and adjustment of therapy: a consensus statement from the American Diabetes Association and the European Association for the Study of Diabetes. Diabetologia. 2009;52(1):17-30.

13. Owen MR, Doran E, Halestrap AP. Evidence that metformin exerts its antidiabetic effects through inhibition of complex 1 of the mitochondrial respiratory chain. Biochem J. 2000;348(Pt 3):607-14.

14. Lee JO, et al. Metformin induces Rab4 through AMPK and modulates GLUT4 translocation in skeletal muscle cells. J Cell Physiol. 2011;226(4):974-81.

15. Fischer $Y$, et al. Action of metformin on glucose transport and glucose transporter GLUT1 and GLUT4 in heart muscle cells from healthy and diabetic rats. Endocrinology. 1995:136(2):412-20.

16. Hundal HS, et al. Cellular mechanism of metformin action involves glucose transporter translocation from an intracellular pool to the plasma membrane in L6 muscle cells. Endocrinology. 1992;131(3):1165-73.

17. Zhou G, et al. Role of AMP-activated protein kinase in mechanism of metformin action. J Clin Invest. 2001;108(8):1167-74.
18. Horton JW. Free radicals and lipid peroxidation mediated injury in burn trauma: the role of antioxidant therapy. Toxicology. 2003;189(1-2):75-88.

19. Gore DC, Rinehart A, Asimakis G. Temporal changes in cellular energy following burn injury. Burns. 2005:31(8):998-1002.

20. Noto $\mathrm{H}$, et al. Cancer risk in diabetic patients treated with metformin: a systematic review and meta-analysis. PLoS One. 2012;7(3):e33411.

21. Evans $\mathbf{J M}$, et al. Metformin and reduced risk of cancer in diabetic patients. Bmj. 2005;330(7503):1304-5.

22. Libby $\mathrm{G}$, et al. New users of metformin are at low risk of incident cancer: a cohort study among people with type 2 diabetes. Diabetes Care. 2009;32(9): $1620-5$

23. Hong J, et al. Effects of metformin versus glipizide on cardiovascular outcomes in patients with type 2 diabetes and coronary artery disease. Diabetes Care. 2013;36(5):1304-11.

24. Dadwal $P$, et al. Activating endogenous neural precursor cells using metformin leads to neural repair and functional recovery in a model of childhood brain injury. Stem Cell Rep. 2015:5(2):166-73.

25. Wang J, et al. Metformin activates an atypical PKC-CBP pathway to promote neurogenesis and enhance spatial memory formation. Cell Stem Cell. 2012; 11(1):23-35

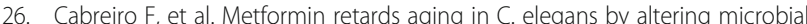
folate and methionine metabolism. Cell. 2013;153(1):228-39.

27. Martin-Montalvo A, et al. Metformin improves healthspan and lifespan in mice. Nat Commun. 2013;4:2192

28. Ashour $A E$, et al. Metformin rescues the myocardium from doxorubicininduced energy starvation and mitochondrial damage in rats. Oxidative Med Cell Longev. 2012;2012:434195.

29. Kobashigawa LC, et al. Metformin protects cardiomyocyte from doxorubicin induced cytotoxicity through an AMP-activated protein kinase dependent signaling pathway: an in vitro study. PLoS One. 2014;9(8):e104888.

30. Cai $\mathrm{H}_{\text {, et }}$ al. Metformin protects the myocardium against isoproterenolinduced injury in rats through alleviating endoplasmic reticulum stress. Pharmazie. 2014;69(1):64-9.

31. Langone $F$, et al. Metformin protects skeletal muscle from cardiotoxin induced degeneration. PLoS One. 2014;9(12):e114018.

32. Lepper C, Partridge TA, Fan CM. An absolute requirement for Pax7-positive satellite cells in acute injury-induced skeletal muscle regeneration. Development. 2011;138(17):3639-46.

33. Fry CS, et al. Regulation of the muscle fiber microenvironment by activated satellite cells during hypertrophy. FASEB J. 2014;28(4):1654-65.

34. Pawlikowski B, et al. Pervasive satellite cell contribution to uninjured adult muscle fibers. Skelet Muscle. 2015;5:42.

35. Amini-Nik S, et al. Pax7 expressing cells contribute to dermal wound repair, regulating scar size through a beta-catenin mediated process. Stem Cells. 2011:29(9):1371-9.

36. Fry CS, et al. Satellite cell activation and apoptosis in skeletal muscle from severely burned children. J Physiol. 2016:594(18):5223-36.

37. Yousuf $Y$, et al. The response of muscle progenitor cells to cutaneous thermal injury. Stem Cell Res Ther. 2017:8(1):234.

38. Finnerty CC, et al. Inducible satellite cell depletion attenuates skeletal muscle regrowth following a scald-burn injury. J Physiol. 2017;595(21): $6687-701$.

39. Gore DC, et al. Influence of metformin on glucose intolerance and muscle catabolism following severe burn injury. Ann Surg. 2005;241(2):334-42.

40. Diao $L$, et al. Alternative mechanism for white adipose tissue lipolysis after thermal injury. Mol Med. 2015;21(1):959-68.

41. Wong W, et al. Surgical approaches to create murine models of human wound healing. J Biomed Biotechnol. 2011;2011:969618.

42. Mestas J, Hughes CC. Of mice and not men: differences between mouse and human immunology. J Immunol. 2004;172(5):2731-8.

43. Barret JP, Jeschke MG, Herndon DN. Fatty infiltration of the liver in severely burned pediatric patients: autopsy findings and clinical implications. J Trauma. 2001;51(4):736-9.

44. Hamrick MW, McGee-Lawrence ME, Frechette DM. Fatty infiltration of skeletal muscle: mechanisms and comparisons with bone marrow adiposity. Front Endocrinol. 2016;7:69.

45. Lin HZ, et al. Metformin reverses fatty liver disease in obese, leptin-deficient mice. Nat Med. 2000:6(9):998-1003.

46. Lingvay I, Raskin P, Szczepaniak LS. Effect of insulin-metformin combination on hepatic steatosis in patients with type 2 diabetes. J Diabetes Complicat. 2007;21(3):137-42. 
47. Calixto MC, et al. Metformin attenuates the exacerbation of the allergic eosinophilic inflammation in high fat-diet-induced obesity in mice. PLoS One. 2013;8(10):e76786.

48. Zhang SQ, et al. Protective effect of metformin on renal injury of C57BL/6 mouse treated with high fat diet. Pharmazie. 2014;69(12):904-8.

49. Holmes BF, Kurth-Kraczek EJ, Winder WW. Chronic activation of 5'-AMPactivated protein kinase increases GLUT-4, hexokinase, and glycogen in muscle. J Appl Physiol (1985). 1999;87(5):1990-5.

50. Fu X, et al. AMP-activated protein kinase stimulates Warburg-like glycolysis and activation of satellite cells during muscle regeneration. J Biol Chem. 2015;290(44):26445-56.

51. Saisho Y. Metformin and inflammation: its potential beyond glucoselowering effect. Endocr Metab Immune Disord Drug Targets. 2015;15(3): 196-205.

52. Lu J, et al. The protective effect and underlying mechanism of metformin on neointima formation in fructose-induced insulin resistant rats. Cardiovasc Diabetol. 2013;12:58

53. Hattori $Y$, et al. Metformin inhibits cytokine-induced nuclear factor kappaB activation via AMP-activated protein kinase activation in vascular endothelia cells. Hypertension. 2006;47(6):1183-8.

54. Li SN, et al. Metformin inhibits nuclear factor kappaB activation and decreases serum high-sensitivity C-reactive protein level in experimental atherogenesis of rabbits. Heart Vessel. 2009;24(6):446-53.

55. Koh SJ, et al. Anti-inflammatory mechanism of metformin and its effects in intestinal inflammation and colitis-associated colon cancer. J Gastroenterol Hepatol. 2014;29(3):502-10.

56. Li H, Malhotra S, Kumar A. Nuclear factor-kappa B signaling in skeletal muscle atrophy. J Mol Med. 2008;86(10):1113-26.

57. Jackman RW, et al. NF-kappaB signaling and transcriptional regulation in skeletal muscle atrophy. Exp Physiol. 2013;98(1):19-24.

58. Mourkioti F, et al. Targeted ablation of IKK2 improves skeletal muscle strength, maintains mass, and promotes regeneration. J Clin Invest. 2006; 116(11):2945-54.

59. Cai D, et al. IKKbeta/NF-kappaB activation causes severe muscle wasting in mice. Cell. 2004;119(2):285-98.

60. Merritt EK, Cross JM, Bamman MM. Inflammatory and protein metabolism signaling responses in human skeletal muscle after burn injury. J Burn Care Res. 2012;33(2):291-7.

61. Cetrone M, Mele A, Tricarico D. Effects of the antidiabetic drugs on the agerelated atrophy and sarcopenia associated with diabetes type II. Curr Diabetes Rev. 2014;10(4):231-7.

62. Tong JF, et al. Metformin mitigates the impaired development of skeletal muscle in the offspring of obese mice. Nutr Diabetes. 2011;1:e7.

63. Mozdziak PE, et al. Hindlimb suspension reduces muscle regeneration. Eur J Appl Physiol Occup Physiol. 1998;78(2):136-40.

64. Paris ND, et al. Smad4 restricts differentiation to promote expansion of satellite cell derived progenitors during skeletal muscle regeneration. Elife. 2016;5.

65. Liu W, et al. Inducible depletion of adult skeletal muscle stem cells impairs the regeneration of neuromuscular junctions. Elife. 2015;4.

66. Horvathy DB, et al. Muscle regeneration is undisturbed by repeated polytraumatic injury. Eur J Trauma Emerg Surg. 2011;37(2):161-7.

67. Sabourin LA, Rudnicki MA. The molecular regulation of myogenesis. Clin Genet. 2000;57(1):16-25.

68. Folmes $C D$, et al. Metabolic plasticity in stem cell homeostasis and differentiation. Cell Stem Cell. 2012;11(5):596-606.

69. Kondoh $\mathrm{H}$, et al. A high glycolytic flux supports the proliferative potential of murine embryonic stem cells. Antioxid Redox Signal. 2007;9(3):293-9.

70. Clarke L, van der Kooy D. Low oxygen enhances primitive and definitive neural stem cell colony formation by inhibiting distinct cell death pathways. Stem Cells. 2009;27(8):1879-86.

71. Eliasson P, Jonsson Jl. The hematopoietic stem cell niche: low in oxygen but a nice place to be. J Cell Physiol. 2010;222(1):17-22.

72. Ochocki JD, Simon MC. Nutrient-sensing pathways and metabolic regulation in stem cells. J Cell Biol. 2013;203(1):23-33.

73. Montarras D, L'Honore A, Buckingham M. Lying low but ready for action: the quiescent muscle satellite cell. FEBS J. 2013;280(17):4036-50.

74. Menendez JA, et al. The Warburg effect version 2.0: metabolic reprogramming of cancer stem cells. Cell Cycle. 2013;12(8):1166-79.

75. Pavlidou T, et al. Metformin delays satellite cell activation and maintains quiescence. Stem Cells Int. 2019;2019:5980465.
76. Ceafalan LC, Popescu BO, Hinescu ME. Cellular players in skeletal muscle regeneration. Biomed Res Int. 2014;2014:957014

77. Hirata A, et al. Expression profiling of cytokines and related genes in regenerating skeletal muscle after cardiotoxin injection: a role for osteopontin. Am J Pathol. 2003;163(1):203-15.

78. Abdullahi A, Amini-Nik S, Jeschke MG. Animal models in burn research. Cell Mol Life Sci. 2014:71(17):3241-55.

79. Hardy D, et al. Comparative study of injury models for studying muscle regeneration in mice. PLoS One. 2016:11(1):e0147198.

80. Viollet B, et al. Cellular and molecular mechanisms of metformin: an overview. Clin Sci. 2012;122(6):253-70.

81. Jørgensen SB, Richter EA, Wojtaszewski JFP. Role of AMPK in skeletal muscle metabolic regulation and adaptation in relation to exercise. J Physiol. 2006; 574(Pt 1):17-31.

82. Jeschke MG, et al. Glucose control in severely burned patients using metformin: an interim safety and efficacy analysis of a phase II randomized controlled trial. Ann Surg. 2016;264(3):518-27.

83. Ragheb $\mathrm{R}$, et al. Free fatty acid-induced muscle insulin resistance and glucose uptake dysfunction: evidence for PKC activation and oxidative stress-activated signaling pathways. Biochem Biophys Res Commun. 2009; 389(2):211-6.

84. Boden $\mathrm{G}$, et al. Mechanisms of fatty acid-induced inhibition of glucose uptake. J Clin Invest. 1994;93(6):2438-46.

85. Dresner $A$, et al. Effects of free fatty acids on glucose transport and IRS-1-associated phosphatidylinositol 3-kinase activity. J Clin Invest. 1999; 103(2):253-9.

86. Lang T, et al. Computed tomographic measurements of thigh muscle crosssectional area and attenuation coefficient predict hip fracture: the health, aging, and body composition study. J Bone Miner Res. 2010;25(3):513-9.

87. Rivas DA, et al. Diminished anabolic signaling response to insulin induced by intramuscular lipid accumulation is associated with inflammation in aging but not obesity. Am J Physiol Regul Integr Comp Physiol. 2016; 310(7):R561-9.

88. Salpeter SR, et al. Risk of fatal and nonfatal lactic acidosis with metformin use in type 2 diabetes mellitus: systematic review and meta-analysis. Arch Intern Med. 2003;163(21):2594-602.

\section{Publisher's Note}

Springer Nature remains neutral with regard to jurisdictional claims in published maps and institutional affiliations.

Ready to submit your research? Choose BMC and benefit from:

- fast, convenient online submission

- thorough peer review by experienced researchers in your field

- rapid publication on acceptance

- support for research data, including large and complex data types

- gold Open Access which fosters wider collaboration and increased citations

- maximum visibility for your research: over $100 \mathrm{M}$ website views per year

At BMC, research is always in progress.

Learn more biomedcentral.com/submissions 